\title{
What does neural plasticity tell us about role of primary visual cortex (V1) in visual awareness?
}

\author{
Juha Silvanto ${ }^{1 *}$ and Geraint Rees ${ }^{2,3 *}$ \\ Brain Research Unit, Low Temperature Laboratory and Advanced Magnetic Imaging Centre, School of Science and Technology, Aalto University, Espoo, Finland \\ 2 Institute of Cognitive Neuroscience, University College London, London, UK \\ 3 Wellcome Trust Centre for Neuroimaging, University College London, London, UK
}

\section{Edited by:}

Morten Overgaard, Aarhus University,

Denmark

\section{Reviewed by:}

Niko Busch, Humboldt Universität Berlin, Germany

Søren Kyllingsbaek, University of

Copenhagen, Denmark

*Correspondence:

Juha Silvanto, Brain Research Unit, Low Temperature Laboratory and Advanced Magnetic Imaging Centre School of Science and Technology, Aalto University, Espoo, Finland. e-mail:silvanto@neuro.hut.fi; Geraint Rees, Institute of Cognitive Neuroscience, University College London, 17 Queen Square, London WC1N 3AR, UK.

e-mail: g.rees@fil.ion.ucl.ac.uk
The complete loss of visual awareness resulting from a lesion to the primary visual cortex (V1) suggests that this region is indispensable for conscious visual perception. There are however a number cases of conscious perception in the absence of $\mathrm{V} 1$ which appear to challenge this conclusion. These include reports of patients with bilateral V1 lesions sustained at an early age whose conscious vision has spontaneously recovered, as well as stroke patients who have recovered some conscious vision with the help of rehabilitation programs. In addition, the phenomenon of hemianopic completion and percepts induced by brain stimulation suggest that V1 may not be necessary for conscious perception in all circumstances. Furthermore, that the visual abilities in the cat are associated with the recovery of normal extrastriate tuning properties rather than emulation of $\mathrm{V} 1$ functions suggests that there is nothing unique about the functional properties of this region in visual awareness. Rather, the dramatic effect of a $\mathrm{V} 1$ lesion on visual awareness may be due to its role in providing the majority of extrastriate visual input, the loss of which abolishes normal neural responsiveness throughout the visual cortex.

Keywords: visual awareness, plasticity, V1, blindsight, transcranial magnetic stimulation, consciousness, extrastriate
A lesion to the primary visual cortex (V1) abolishes all conscious visual perception in the corresponding part of the visual field. However, the ability of some patients to unconsciously detect and discriminate stimuli within the field defect (see Cowey, 2010 for reviews of "blindsight"), implies that the loss of perceptual experience is not simply due to information failing to reach the visual system, and strongly implicate $\mathrm{V} 1$ in visual awareness. The role of $\mathrm{V} 1$ in determining the content of subjective awareness is also supported by studies carried out in neurologically normal observers (see Tong, 2003; Ro, 2010 for reviews).

There are however a number cases of conscious visual perception in the absence of V1 which appear to challenge the view that this region plays an indispensable role in visual awareness. These include reports of children born without $\mathrm{V} 1$, stroke patients who have recovered some conscious vision with the help of rehabilitation programs, as well as the phenomenon of hemianopic completion and percepts induced by brain stimulation. Such cases are important to the study of neural correlates of consciousness (NCC): if conscious perception of a given attribute is initially abolished by a lesion but subsequently recovers, this indicates that the NCC of that attribute is not fixed to the affected brain region. In this review we will consider the implications of plasticity to understanding the role of $\mathrm{V} 1$ in visual awareness.

\section{CONSCIOUS PERCEPTION AFTER A BILATERAL V1 LESION}

For the present discussion, the critical question is the extent to which visual awareness can recover in the complete absence of $\mathrm{V} 1$, as this would indicate that V1 is not indispensable for awareness. Cases of bilateral V1 lesion are thus of paramount interest. However, reports of complete bilateral lesions of occipital cortex are rare, as in many cases some occipital cortex may remain functional, which complicates interpretation. For example, Bova et al. (2008), reported a patient who suffered bilateral occipital lobe infarction at the age of 2 years 6 months. In the acute phase, his visual behavior was consistent with complete bilateral visual loss; fixation was absent, there was no blink response to threat, and he was only able to recognize objects and family members using compensatory (touch and auditory) abilities. However, 18 months after the lesion, he could see and reach small objects on the ground, name colors, and recognize himself and his relatives even in photographs. At 6 years 8 months, visual recognition acuity was $10 / 10$ in both eyes and neuro-ophthalmological examination was normal, except for persistence of the visual field defect in the upper hemifield and a selective impairment for complex visuospatial skills. This case appears to suggest that conscious perception can almost fully recover in the absence of V1. However, MRI scan suggested a partial sparing of the striate cortex, and this was confirmed by the presence of some residual occipital response of the VEPs, suggesting the presence of some functional striate cortex. Therefore, while the presence of some residual V1 may not fully explain conscious visual perception in this patient, it may have facilitated recovery (see, e.g., Payne and Rushmore (2003)) and complicates the implications of this study for understanding the role of V1 in visual awareness. The vast majority of V1 lesions are incomplete, and thus the number of cases from which strong theoretical conclusions can be drawn is limited.

There are however few cases in which the absence of early visual cortex has been verified. Amicuzi et al. (2006) reported the case of a 5-year-old girl in whom ultrasonography 16 days after birth showed an enlargement of the occipital horns of the lateral ventricles, mostly on the left side. At the age of 5, MRI scans disclosed an 
absence of occipital pole bilaterally, as well as of occipitotemporal regions in the right hemisphere and occipitoparietal regions in the left hemisphere. Functional examinations revealed that basic visual abilities such as visual acuity, contrast sensitivity and visual field were present although somewhat compromised. On visual perceptual assessment, a selective impairment of figure-ground segregation was found, whereas color, form, and orientation discrimination were almost normal. Visual recognition impairment was present for objects, faces, actions, and scenes. While the neural correlates of conscious vision in this subject are unclear due to the lack of neuroimaging evidence, it is clear that it must be the remaining extrastriate cortex which enables conscious perception to arise. This was supported by diffusion tensor imaging (DTI) evidence (Amicuzi et al., 2006) which highlighted connections from lateral geniculate nucleus to the intact extrastriate cortex which bypass the damaged areas. Expansion of pathways that can bypass $\mathrm{V} 1$ and directly connect subcortical nuclei with extrastriate visual structures is believed to be critical in neural plasticity following the loss of V1 and may also mediate blindsight (see, e.g., Cowey, 2004; Payne and Lomber, 2002).

A second intriguing case involves a 21 -year-old male with extensive bilateral damage present since birth (Giaschi et al., 2003). This case is highly informative because the absence of the striate cortex was confirmed with anatomical MRI scans as well as using and functional MRI and high-resolution EEG. Furthermore, the neural basis of conscious perception was investigated using fMRI.

The visual impairment, likely to be due to hypoxic brain insult at birth, was severe. At the age of 2 years, he was diagnosis of congenital cortical visual impairment. Full-field flash stimuli evoked no potentials from the occipital cortex and no alpha rhythm and or responses on eye opening and closure was seen in EEG. When examined at the age of 21 , he could identify a few simple shapes, by scanning them repeatedly close to his eyes. The patient was able to name the colors of large stimuli, but he made many errors in color discrimination on the Quantitative Color Vision Test. He could appreciate size differences and count coins, without touch, only when they were widely separated. The patients was however able to detect stimuli moving at high speeds; at low speeds (below $3.5^{\circ} \mathrm{s}$ ), his accuracy fell to chance performance. Importantly, he reported that he could "see" the motion, indicating that his motion detection ability was not blindsight; his detection ability was accompanied by phenomenal awareness. The neural basis of this motion perception was investigated with fMRI. Although the motion-selective area V5/ MT appeared to be anatomically intact, no functional activation was observed in this region. Instead, fast radial motion activated regions in right premotor cortex, right medial posterior cingulate, right medial precuneus, left anterior superior temporal gyrus, and left and right posterior superior temporal sulcus. No activation was induced by the slow stimuli.

This case is interesting for several reasons. Firstly, although conscious residual vision for motion in the absence of awareness of other stimulus attributes has been previously reported (Riddoch, 1917), this is the only reported case in which the absence of striate cortex has been verified. Secondly, this case provides both anatomical and neuroimaging evidence indicating that some visual perception is possible in the complete absence of V1; no striate cortex tissue was visible on anatomic MRI scan, and no evoked responses were found with EEG or fMRI. Thirdly, it appears that parietal and temporal regions gave rise to conscious perception in this subject.

\section{PLASTICITY FOLLOWING UNILATERAL V1 LESIONS}

While the evidence from bilateral occipital lesions demonstrates that conscious vision can recover in the complete absence of $\mathrm{V} 1$, the neural basis of that recovery is unclear. More is known about the development of new visual field maps accompanying visual function recovery after unilateral V1 lesions. Muckli et al. (2009) report a subject whose development of the right cerebral hemisphere terminated before the 7th week of embryonic gestation. Despite the complete loss of right hemisphere at birth, the patient had close to normal vision in both hemifields and successfully mastered activities requiring bilateral coordination such as roller skating and bike riding. fMRI mapping revealed that the patient's remaining hemisphere contained visual field representations of not only he contralateral visual hemifield (as is the case in neurologically normal development) but, surprisingly, also maps of the ipsilateral (left) visual hemifield: islands of ipsilateral visual field representations were located along the representations of the vertical meridian. In $\mathrm{V} 1$, smooth and continuous maps from contra- and ipsilateral hemifield overlap each other, whereas in ventral V2 and V3 ipsilateral quarter field representations invaded small distinct cortical patches. This is significant because normal subjects do not show ipsilateral representations in early visual areas.

Brain plasticity tends to be more evident after congenital or early acquired brain injury (see, e.g., Werth, 2008; Guzzetta et al., 2010 for reviews). While potential for plasticity is greatly reduced in adulthood, the fact that visual field rehabilitation protocols have at least limited success in shrinking visual field defects (e.g., Zihl and von Cramon, 1986; Kerkhoff et al., 1994; Kasten et al., 1997; Schmielau and Wong, 2007) indicates that some functionally significant neural plasticity does occur. Furthermore there is also electrophysiological evidence of plastic changes in the adult monkey visual cortex. For example, after a few months of wearing prisms that laterally reversed the visual field, neurons in the macaque V1 respond to both ipsi- as well as to contralateral visual fields (Sugita, 1996).

The link between neural plasticity and functional recovery of conscious vision was recently investigated by Henriksson et al. (2007), who trained a 61-year-old patient with homonymous hemianopia with flicker stimulation. The training was performed 3 years after the stroke, at a stage in which the subject showed a stable homonymous hemianopia, with no evoked neuromagnetic responses in response to visual stimulation of his blind hemifield. During training, the patient became conscious of stimuli presented in the blind hemifield; form vision emerged, and the far periphery of the blind hemifield brightened. Changes in neural responsiveness during training were documented with magnetoencephalography, and the cortical organization after training was examined with fMRI. The key finding was that, after training, visual information from both hemifields was processed mainly in the intact hemisphere. Specifically, fMRI mapping revealed a representation of both the blind and the normal hemifields in the same set of cortical areas in the intact hemisphere, more specifically in the visual motion-sensitive area V5/MT, in a region around the superior temporal sulcus and in retinotopic visual areas V1, V2, V3, and V3a. 
This case differs from that reported by Muckli et al. (2009) in that restored function and the coinciding involvement of the contralesional hemisphere were due to therapeutic intervention in an elderly patient rather than spontaneous recovery occurring in early life. Nevertheless, there is a remarkable similarity between them: in both cases, conscious vision appears to be associated with the development of ipsilateral visual field representations in the early visual cortex (although the extent to which visual recovery depends particularly on V1 is not known). It thus appears that, as long as some functional V1 remains, it can participate in the mediation of visual awareness from spatial positions that it did not encode prior to the lesion. These unilateral cases are thus not inconsistent with the view that retinotopic representation of the visual field in V1 plays a necessary role in visual awareness.

\section{PHENOMENAL AWARENESS IN BLINDSIGHT}

In the above examples, at least some visual awareness was possible after a V1 lesion. However, in most patients visual information fails to reach awareness although it can in some circumstances unconsciously detected. Perhaps the most frequently studied blindsight subject is GY, who has a large unilateral lesion in the left medial occipital lobe, caused by a traffic accident at the age of 8 . Striate cortex is absent in the left hemisphere, except at the occipital pole corresponding to about three to four degrees of macular sparing. Although GY (and other blindsight patients) perform well in numerous visual tasks (see Stoerig and Cowey, 1997 for reviews), there is no phenomenal awareness associated with these functions in the blind field.

Interestingly, unlike in the subjects reported by Muckli et al. (2009) and Henriksson et al. (2007), V1 in GY's intact hemisphere is not activated by stimuli presented in the blind field that induce blindsight. In contrast, extrastriate areas in the damaged as well as intact hemisphere (such as V5/MT) do show BOLD responses to stimuli presented in the blind field. However, these extrastriate activations seem to be unable to give rise to conscious perception in the absence of V1. Taken together with the findings of Henriksson et al. (2007), it seems that after a unilateral $\mathrm{V} 1$ lesion, conscious vision tends to primarily recover through the development of retinotopic representations of the affected field in the contralesional V1. Representations of the blind field in the extrastriate (either in the intact or damaged hemisphere) do not seem to enable awareness to arise, at least when the lesion is sustained in adulthood.

There are however circumstances in which hemianopic patients with a V1 lesion sustained in adulthood can experience qualia in the blind field. An example of this is hemianopic completion, which refers to the perceptual completion of figures located across the vertical meridian in the context of hemianopia, such that one half of the figure falls within the blind hemifield (e.g., Bender and Kahn, 1949). Marcel (1998) used after-images to demonstrate veridical conscious perception of shape in GY's blind field when it was accompanied by a shape in the sighted field. When only the normal hemifield was exposed to visual stimulation, an afterimage appeared in the normal hemifield; in contrast, when the blind field was stimulated, no afterimage was perceived. Critically, when both the normal and the blind hemifields were stimulated in combination, such that the formed a good Gestalt, the after-image appeared in both hemifields. This result shows that visual stimulation of GY's blind field can give rise to visual experiences, if the blind field stimulus is part of a larger bilateral stimulus.

The neural basis of such completion has been recently studied by Weil et al. (2009) in a patient with a homonymous hemianopia following occipital stroke that was associated with hemianopic perceptual completion across the blind field. Completion was found to be associated with activation anterior to retinotopic cortex in the lingual gyrus in the right occipital cortex, contralateral to the lesion, ipsilateral to the illusory edge of the stimulus. This region was located in visually responsive ventral visual cortex near to reported coordinates for the human lateral occipital complex (Avidan et al., 2002). Importantly, activity in the early visual cortical areas V1-V3 was not associated with completion, demonstrating that visual awareness does not always depend on activity in V1.

A further example of conscious perception without the development of an ipsilateral visual field representation in the intact V1 has come from studies using transcranial magnetic stimulation (TMS). When TMS is applied over the extrastriate visual areas, neurologically normal observers perceive flashes of light (phosphenes). However, when TMS is applied unilaterally over presumably intact extrastriate areas in GY' damaged hemisphere, he does not perceive phosphenes; this is consistent with the view that extrastriate activation cannot reach awareness in the absence of V1. However, when TMS was applied over the extrastriate area V5/MT in both the damaged and intact hemisphere in close temporal proximity, GY perceived bilateral phosphenes that extend into the blind hemifield. Using this technique, GY could even experience colored percepts in his blind field (Silvanto et al., 2008).

At the perceptual level, TMS-induced bilateral phosphenes seem similar to hemianopic completion, and it may be that they are mediated by the intact extrastriate cortex via callosal connections. Consistent with this view, a DTI study by Bridge et al. (2008) found a substantial cortico-cortical connection in GY between V5/MT bilaterally, consistent with the possibility that information can be transferred from the damaged to the intact hemisphere. While the neural basis of these blind field phosphene is unclear, the critical point is that GY's intact V1 cannot be directly involved as it does not contain a retinotopic representation of the blind visual field. The main implication of these finding therefore is that a retinotopic representation of the visual field in V1 cannot be necessary for phenomenal awareness.

\section{WHAT DOES PLASTICITY TELL ABOUT THE ROLE OF V1 IN AWARENESS?}

The evidence can be summarized as follows: after a unilateral V1 lesion, spontaneous or training-induced plasticity can lead to the development of ipsilateral visual field maps in the unaffected V1, in both children and adults. The functional recovery resulting from spontaneous plasticity is robust in childhood, with visual functions being almost normal (Muckli et al., 2009). When the lesion occurs in adulthood after the visual system has fully developed, the extent of visual recovery is much smaller, and the development of new visual field maps in the intact V1 may require behavioral rehabilitation programs (Henriksson et al., 2007). These cases support the view that V1 is necessary for visual awareness, as the development of ipsilateral visual field maps in the intact early visual cortex appears to be necessary for the recovery of conscious vision. 
Importantly however, the cases in which V1 is missing bilaterally (Giaschi et al., 2003; Amicuzi et al., 2006) demonstrate that plastic changes which enable some conscious perception to recover do not necessarily require $\mathrm{V} 1$. While visual functions may remain compromised, the fact that conscious vision can develop at all indicates that the brain does not need V1 to give rise to conscious visual percepts. Finally, the phenomena of hemianopic completion (e.g., Bender and Kahn, 1949; Marcel, 1998; Weil et al., 2009) and blind field phosphene perception (Silvanto et al., 2007, 2008) also demonstrate that that some conscious vision is possible without the development of an ipsilateral visual field representation in the contralesional V1. While the extent to which plasticity plays role in these latter phenomena is not clear, they are nevertheless inconsistent with the view that a retinotopic representation of the visual field in V1 is a prerequisite of all visual awareness.

\section{ARE FUNCTIONAL PROPERTIES OF V1 NECESSARY FOR AWARENESS?}

A brain recovering from lesions sustained early in life have undergone a very different developmental path than that of a neurologically normal subject, and one might question the usefulness of referring in this context to visual areas which are found in normally developed visual cortex. While this may be true, the other side of the coin is that there appears to be nothing unique about specific anatomically defined areas such as V1 in enabling conscious perception. Of course, what might make V1 necessary for awareness may not be its anatomical position at the back head, but rather, the functional properties and anatomical connectivity of its neurons. If we define V1 not in terms of its anatomical location as the portion of the brain at the occipital pole around the calcarine sulcus, but rather in terms of the functional properties of its neurons, the question becomes: is the recovery of conscious perception a consequence of unaffected cortex taking over the functional properties of V1? If so, this would suggest that V1 (in its functional definition) is indeed necessary for visual awareness.

It is impossible to offer a conclusive answer to this question, but electrophysiological evidence obtained in the cat may offer some clues. Neurons in posteromedial lateral suprasylvian area, an extrastriate visual area in the cat, lose neural tuning after a damaged to the visual cortex. However, neural responsiveness can recover, especially if the lesion has been sustained in childhood (e.g., Spear and Baumann, 1979; Tong et al., 1984). The important question is whether this recovery leads to neurons developing the response properties of the damaged cortex. This question has been addressed in a number of studies (e.g., Maffrei and Fiorentini, 1973; Movshon et al., 1978; Guido et al., 1992). The key finding is that the spatial or temporal response properties in this region are not different from those found in normal cats, and they are unlike those of normal striate cortex cells. In other words, the outcome of neural plasticity is the development of normal tuning properties. That these neurons did not take over the neural properties of the damaged cortex suggests visual recovery in the cat does not rely on V1 functions being emulated by other brain areas.

That the functional properties of V1 are not emulated by other areas does not imply that the functional role of $\mathrm{V} 1$ in a given visual skill cannot be taken over by the unaffected cortex. Indeed, there is evidence that visual abilities that are normally based on V1 activity may rely on other areas after a V1 lesion (see Payne and Lomber,
2002). However, this does imply a change in neural tuning - a visual area with somewhat similar neural tuning as a normal V1 may take over the role. Consequently, the particular visual ability may be impaired because the neural properties in that region may be less suited for this purpose than those in the normal V1.

Interestingly, neural tuning in the extrastriate cortex of the monkey shows rather less recovery after a V1 lesion. Rodman et al. (1989) found weak responses to visual stimuli in V5/MT (with only 5\% of neurons responding strongly to visual stimulation), and receptive fields were difficult to localize precisely. A different conclusion was reached by Girard et al. (1992), who used cooling to deactivate V1 and found that $80 \%$ of neurons remained responsive to visual stimulation. However, the cooling plate did not cover the whole of V1 (affecting only approximately $4^{\circ}$ of the lower quadrant); thus the majority of V5/MT neurons did not lose their V1 input, which could explain their high level of responsiveness. Subsequent studies (e.g., Azzopardi et al., 2003; Collins et al., 2003) on monkeys found no or severely reduced neural responsiveness in V5/MT. For example, in the study by Azzopardi et al. (2003) the neural responses to moving stimuli in V5/MT were as small as the V5/MT responses to static stimuli in the normal brain. More recently, functional MRI in monkeys demonstrates weak responses of extrastriate cortex to visual stimulation following a chronic V1 lesion, with overall activity evoked by visual stimulation approximately one-fifth of normal levels (Schmid et al., 2010). Interestingly, this residual activity was critically dependent on the lateral geniculate nucleus as it disappeared when that structure was inactivated. It remains possible however that in monkeys with V1 damage in infancy can develop more normal neural selectivity in extrastriate cortex, as there is significantly more residual vision compared to monkeys with lesions sustained in adulthood (Moore et al., 1996). Nevertheless, the electrophysiological evidence from lesioned monkeys suggests that in the absence of V1, the functioning of the extrastriate cortex is severely compromised.

In summary, while animal studies cannot conclusively answer the question of whether the recovery of visual awareness requires the development of functional properties of a normal V1 in another brain area, they suggest that this is not required. Rather, it appears that as long as extrastriate areas have normal functionality, visual perception can arise.

\section{CONCLUSION}

The objective of this review was to consider the implications of neural plasticity for understanding the role of $\mathrm{V} 1$ in visual awareness. Although a lesion to this region abolishes all phenomenal awareness, conscious vision can recover; the extent of this recovery appears to be linked to the age at which the lesion was sustained. The ability of the visual system to create conscious visual percepts in the absence of $\mathrm{V} 1$ suggests that there is fundamentally nothing unique about specific anatomically defined areas such as V1 in enabling conscious perception. Furthermore, that the extrastriate neural plasticity enabling visual recovery in the cat is associated with the recovery of normal tuning properties rather than emulation of functional properties of V1 neurons suggests that there is nothing unique about the neural selectivity of this region in visual awareness. Rather, the dramatic effect of a V1 lesion on visual awareness may be due to its role as the provider of input to most of extrastriate cortex that enables normal extrastriate neural responsiveness. 


\section{REFERENCES}

Amicuzi, I., Stortini, M., Petrarca, M., Di Giulio, P., Di Rosa, G., Fariello, G., Longo, D., Cannatà, V., Genovese, E., and Castelli, E. (2006). Visual recognition and visually guided action after early bilateral lesion of occipital cortex: a behavioral study of a 4.6-yearold girl. Neurocase 12, 263-279.

Avidan, G., Harel, M., Hendler, T., BenBashat, D., Zohary, E., and Malach, R. (2002). Contrast sensitivity in human visual areas and its relationship to object recognition. J. Neurophysiol. 87, 3102-3116

Azzopardi, P., Fallah, M., Gross, C. G., and Rodman, H. R. (2003). Response latencies of neurons in visual areas MT and MST of monkeys with striate cortex lesions. Neuropsychologia 41, 1738-1756.

Bender, M. B., and Kahn, R. L. (1949). After-imagery in defective fields of vision. J. Neurol. Neurosurg. Psychiatr. 12, 196-204.

Bova, S. M., Giovenzana, A., Signorini, S., La Piana, R., Uggetti, C., Bianchi, P. E., and Fazzi, E. (2008). Recovery of visual functions after early acquired occipital damage. Dev. Med. Child Neurol. 50, 311-315.

Bridge, H., Thomas, O., Jbabdi, S., and Cowey, A. (2008). Changes in connectivity after visual cortical brain damage underlie altered visual function. Brain 131(Pt 6), 1433-1444.

Collins, C. E., Lyon, D. C., and Kaas, J. H. (2003). Response of neurons in the middle temporal visual area after long-standing lesions of the primary visual cortex in the adult new world monkeys. J. Neurosci. 23, 2251-2264.

Cowey, A. (2004). The 30th Sir Frederick Bartlett lecture. Fact, artefact, and myth about blindsight. Q. J. Exp. Psychol. A. 57, 577-609.

Cowey, A. (2010). The blindsight saga. Exp. Brain Res. 200, 3-24.

Giaschi, D., Jan, J. E., Bjornson, B., Young, S. A., Tata, M., Lyons, C. J., Good, W. V., and Wong, P. K. (2003). Conscious visual abilities in a patient with early bilateral occipital damage. Dev. Med. Child Neurol. 45, 772-781.

Girard, P., Salin, P. A., and Bullier, J. (1992). Response selectivity in area MT of the macaque monkey during reversible inactivation of area V1. J. Neurophysiol. 67, 1437-1446.

Guido, W., Spear, P. D., and Tong, L. (1992). How complete is physiological compensation in extrastriate cortex after visual cortex damage in kittens. Exp. Brain Res. 91, 455-466.

Guzzetta, A., D’Acunto, G., Rose, S. Tinelli, F., Boyd, R., and Cioni, G. (2010). Plasticity of the visual system after early brain damage. Dev. Med. Child Neurol. doi: 10.1111/j.14698749.2010.03710.x. [Epub ahead of print].

Henriksson, L., Raninen, A., Näsänen, R., Hyvärinen, L., and Vanni, S. (2007). Training-induced cortical representation of a hemianopic hemifield. J. Neurol. Neurosurg. Psychiatry 78, 74-81.

Kasten, E., Strasburger, H., and Sabel, B. A. (1997). Programs for diagnosis and therapy of visual field deficits in vision rehabilitation. Spat. Vis. 10, 499-503.

Kerkhoff, G., Münssinger, U., and Meier, E. K. (1994). Neurovisual rehabilitation in cerebral blindness. Arch. Neurol. 51, 474-481.

Maffrei, L., and Fiorentini, A. (1973). The visual cortex as a spatial frequency analyser. Vision Res. 13, 1255-1267.

Marcel, A. J. (1998). Blindsight and shape perception: deficit of visual consciousness or of visual function? Brain 121 1565-1588.

Moore, T., Rodman, H. R., Repp, A. B., Gross, C. G., and Mezrich, R. S. (1996). Greater residual vision in monkeys after striate cortex damage in infancy. J. Neurophysiol. 76, 3928-3333.

Movshon, J. A., Thompson, I. D., and Tolhurst, D. J. (1978). Spatial and temporal contrast sensitivity of neurons in areas 17 and 18 of the cat's visual cortex. J. Physiol. 283, 101-120.

Muckli, L., Naumer, M. J., and Singer, W. (2009). Bilateral visual field maps in a patient with only one hemisphere. Proc. Natl. Acad. Sci. U.S.A. 106 13034-13039.

Payne, B. R., and Lomber, S. G. (2002). Plasticity of the visual cortex after injury: what's different about the young brain? Neuroscientist 8, 174-185.

Payne, B. R., and Rushmore, R. J. (2003). Animal models of cerebral neglect and its cancellation. Neuroscientist. 9, 446-454.

Riddoch, G. (1917). Dissociation of visual perceptions due to occipital injuries, with especial reference to appreciation of movement. Brain 40, 15-57.

Ro, T. (2010). What can TMS tell us about visual awareness? Cortex 46, 110-113.

Rodman, H. R., Gross, C. G., and Albright, T. D. (1989). Afferent basis of visual response properties in area MT of the macaque. I. Effects of striate cortex removal. J. Neurosci. 9, 2033-2050.

Schmid, M. C., Mrowka, S. W., Turchi, J., Saunders, R. C., Wilke, M., Peters, A. J., Ye, F. Q., and Leopold, D. A (2010). Blindsight depends on the lateral geniculate nucleus. Nature 466, 373-377.

Schmielau, F., and Wong, E. K. Jr. (2007) Recovery of visual fields in brain-lesioned patients by reaction perimetry treatment. J. Neuroeng. Rehabil. 4, 31 .

Silvanto, J., Cowey, A., Lavie, N., and Walsh, V. (2007). Making the blindsighted see. Neuropsychologia 45 , 3346-3350.

Silvanto, J., Cowey, A., and Walsh, V. (2008). Inducing conscious perception of colour in blindsight. Curr. Biol. 18 , R950-R951.

Spear, P. D., and Baumann, T. P. (1979) Effects of visual cortex removal on receptive field properties of neurons in the lateral suprasylvian visual area of the cat. J. Neurophysiol. 42(Pt. 1), 31-56.

Stoerig, P., and Cowey, A. (1997). Blindsight in man and monkey. Brain 120(Pt 3), 535-559 (review).

Sugita, Y. (1996). Global plasticity in adult visual cortex following reversal of visual input. Nature 380, 523-526.
Tong, F. (2003). Primary visual cortex and visual awareness. Nat. Rev. Neurosci. 4, 219-229.

Tong, L., Kalil, R. E., and Spear, P. D. (1984). Critical periods for functional anatomical compensation in the lateral suprasylvian visual area following removal of visual cortex in cats. J. Neurophysiol. 52, 941-960.

Weil, R. S., Plant, G. T., James-Galton, M. and Rees, G. (2009). Neural correlates of hemianopic completion across the vertical meridian. Neuropsychologia 47, 457-464.

Werth, R. (2008). Cerebral blindness and plasticity of the visual system in children. A review of visual capacities in patients with occipital lesions, hemispherectomy or hydranencephaly. Restor. Neurol. Neurosci. 26, 377-389.

Zihl, J., and von Cramon, D. (1986). Visual field rehabilitation in the cortically blind? J. Neurol. Neurosurg. Psychiatry 49, 965-966.

Conflict of Interest Statement: The authors declare that the research was conducted in the absence of any commercial or financial relationships that could be construed as a potential conflict of interest.

Received: 22 November 2010; paper pending published: 16 December 2010; accepted: 04 January 2011; published online: 20 January 2011.

Citation: Silvanto J and Rees G (2011)

What does neural plasticity tell us about role of primary visual cortex (V1) in visual awareness? Front. Psychology 2:6. doi: 10.3389/fpsyg.2011.00006

This article was submitted to Frontiers in Consciousness Research, a specialty of Frontiers in Psychology.

Copyright (C) 2011 Silvanto and Rees. This is an open-access article subject to an exclusive license agreement between the authors and Frontiers Media SA, which permits unrestricted use, distribution, and reproduction in any medium, provided the original authors and source are credited. 